
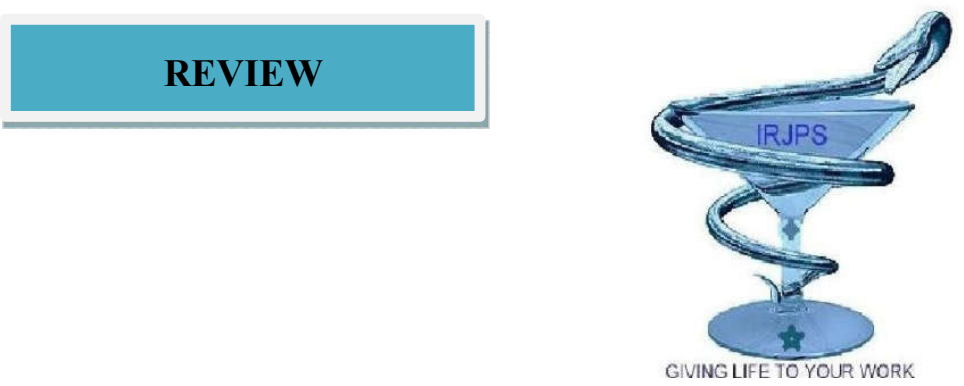

\title{
A REVIEW ON THE SIGNIFICANCE OF MICROBUBBLES
}

\author{
Shayeri Chatterjee ${ }^{1}$, Ramesh Kumari Dasgupta ${ }^{2}$ \\ ${ }^{1}$ Calcutta Institute of Pharmaceutical Technology and Allied Health Sciences, Banitabla, Uluberia, Howrah 711316 \\ ${ }^{2}$ Bharart Technology, Banitabla, Uluberia, Howrah.
}

\section{Submitted on: 27.03.18; $\quad$ Revised on: 14.04.18; $\quad$ Accepted on: 16.04.18}

\begin{abstract}
The review on the significance of microbubbles is about the size, preparation, drug loading methods and uses of microbubbles in various fields like pharmaceutical, biotechnology, diagnostic and research applications. The size of the microbubbles ranges between one micrometer and one millimeter. They are used in medical diagnostics as a contrast agent for ultrasound imaging. The gas-filled microbubbles oscillate and vibrate, when a sonic energy field is applied and may reflect ultrasound waves. This distinguishes the microbubbles from surrounding tissues. Microbubbles must be encapsulated with a solid shell which is made from either a lipid or a protein such as Optison microbubbles consisting of perfluoropropane gas, encapsulated by serum albumin shell. Microbubbles are used to carry a drug or gene to a specific area of interest and where the ultrasounds burst them, causing site-specific delivery of the bioactive materials. Furthermore, the ability of albumin-coated microbubbles to adhere to vascular regions with glycocalix damage or endothelial dysfunction is another possible mechanism to deliver drugs even in the absence of ultrasound. Its scope extends beyond conventional microbubbles to all other small particulate systems such as self assembling structures that involve preparative manipulation.
\end{abstract}

KEY WORDS: Microbubbles, Diagnostics, Ultrasound.

Corresponding Author: Shayeri Chatterjee

E-mail:- minudasgupta007@gmail.com
Indian Research Journal of Pharmacy and Science; 16(2018)1319-1323;

Journal Home Page: https://www.irjps.in DOI: 10.21276/irjps.2018.5.1.10 


\section{INTRODUCTION}

There has been a significant progress in the field of colloidal bubbles or microbubbles and its utilization in targeted drug delivery. Microbubbles are microscopic, gas filled bubbles used in the pharmaceutical industries to image blood flow, dissolve blood clots and diagnostic etc. Owing to its extremely small size ((less than $\sim 10 \mu \mathrm{m}$ diameter), it displays similar rheology in the capillaries and blood vessels, inside the body. Gas bubbles of this size in aqueous media are inherently unstable owing to surface tension effects, and therefore require a stabilizing shell. The shell may be composed of surfactants, lipids, proteins, polymers, or a combination of these materials. Because the interior gas is a poor solvent for drug molecules, loading strategies must be employed within or onto the shell ${ }^{1}$.

Two possible strategies for delivering drugs and genes with microbubbles are emerging. The first consists on the ultrasound-mediated microbubbles destruction, which is based on the cavitation of microbubbles induced by ultrasound application, and the second is the direct delivery of substances bound to microbubbles in the absence of ultrasound. Different drugs and genes can be incorporated into the ultrasound contrast agents. It has already been demonstrated that perfluorocarbon-filled albumin microbubbles avidly bind proteins and synthetic oligonucleotide. In a similar way, microbubbles can directly take up genetic material, such as plasmids and adenovirus and phospholipid-coated microbubbles have a high affinity for chemotherapeutic drugs. Furthermore, specific legands for endothelial cell adhesion molecules, such as pselectin and leukocyte intercellular adhesion molecule 1 (ICAM-1), can be attached to both lipid and albumin-encapsulated microbubbles, which increases their deposition to activated endothelium ${ }^{2}$. In this review an overview on microbubbles shell materials, mechanism of release through the microbubbles shell wall and / or desorption from carrier, techniques of preparation, many uses to which microbubbles are included. .

\section{TYPES OF SHELLS USED FOR THE MICROBUBBLES}

\subsection{PROTEIN SHELLS:}

Protein-based microbubbes form relatively rigid shells by disulfide bridging of proteins that surround and stabilize the gas core of the microbubbles. These microbubbles formulations are commonly are made by using albumin proteins. Incorporation of other bioactive proteins, or two possible strategies for delivering drugs and genes with microbubbles are emerging. The microbubble protein shell can potentially improve the functionality of microbubbles and significantly enhance their importance in biomedical applications. The first albumin microbubble formulation was approved by the US Food and Drug Administration (FDA) was Albunex (GE Healthcare). An Albunex suspension consists of roughly $7 \times 10^{8}$ microbubbles $/ \mathrm{mL}$ with a size range from 1 to $15 \mu \mathrm{m}$ diameter. Albunex is stable upon refrigeration for at least two years ${ }^{3}$.

\subsection{LIPID SHELLS:}

Lipid-based microbubbles are formed by selfassembly of phospholipids into a monolayer at the gas-water interface of the microbubbles, which are held together by weak hydrophobic and van der's Waals interactions. The thin monolayer of the lipidbased microbubbles makes it highly responsive to ultrasound which can be advantages for both molecular imaging (exposing buried ligands) and drug/gene delivery ${ }^{4}$.

\subsection{POLYMER SHELLS:}

Polymer-based microbubbles consist of thick bulky shells formed by cross linking and entanglement of polymer chains. Polymer-based microbubbles can be fabricated using a wide variety of biocompatible materials which allows more versatility in their design. Polymer-based microbubbles are very stable and exhibit a severely dampened response in an acoustic field. These are stabilized by a thick shell comprising cross-linked or entangled polymeric 
species. The bulk nature of the polymer shell makes it more resistant to area compression and expansion than its lipid and albumin counterparts, which reduces the echogenicity and drug delivery activity. For example, polymer microbubbles have been observed to fracture during insonification, thereby releasing their gas core via extrusion through the shell defect. The resulting gas bubble was unstable and rapidly dissolved according to the classical Epstein and Plesset equation. The shell, on the other hand, remained intact and often propelled away from the gas core; this ballistic effect may be useful for drug delivery. However, they are less commonly used for drug and gene delivery applications ${ }^{5}$

\subsection{SURFACTANT SHELLS:}

The mixture of synthetic surfactants SPAN-40 and TWEEN-40 were used to make the microbulles to form a more stable film. The SPAN/TWEEN solution were sonicated in the presence of air to form stable microbubbles. By using a Langmuir trough, they were able to establish the correct ratio of SPAN to TWEEN (roughly 1:1) to use for maximum film stability. Interestingly, its shown that surfactant derived from sonicated microbubbles were more stable. They were capable of reaching higher collapse pressures on the Langmuir trough. Indicating that, the sonication process modified the surfactant to form a more stable film, the microbubbles stabilized by mixtures of the synthetic surfactants SPAN-40 and TWEEN $-40^{5}$.

\subsection{POLY ELECTROLYTE MULTILAYER SHELLS:}

It involves polyelectrolyte multilayer (PEM) shells on preformed microbubbles. The preformed microbubbles are coated with a charged surfactant or protein layer, which serves as a substrate for PEM deposition. The layer-by-layer assembly tec hnique is used to sequentially adsorb oppositely charged polyions to the microbubble shell. Shchukin et al. were the first to report PEM deposition onto microbubbles. They used the polymers poly (ally amine hydrochloride) (PAH) and poly(styrene sulfonate) (PSS) for the polyion pair. This system gave a relatively uniform PEM coating that provided the microbubbles with remarkable stability ${ }^{6}$.

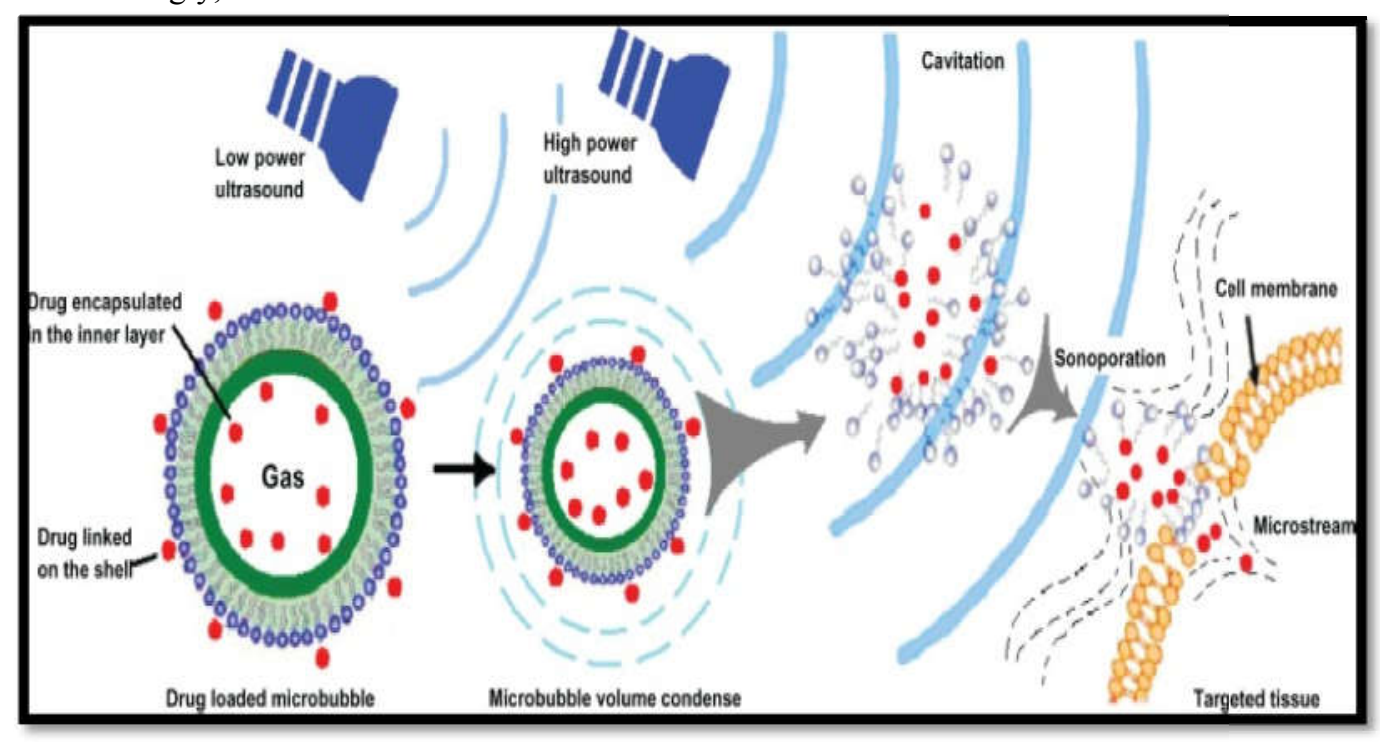

Fig. 1 Microbubbles in Target drug Delivery System

\section{METHOD OF PREPRATION OF MICROBUBLES}

Albumin-coated microbubbles are formed by sonication of a heated solution of $5 \%(\mathrm{w} / \mathrm{v})$ human serum albumin in the presence of air (Fig.1). During sonication, microbubbles of air are formed which become encapsulated within a $15-\mathrm{nm}$ thick shell of aggregated albumin. Heating is necessary to denature the albumin prior to sonication and facilitate encapsulation. Biochemical analysis suggested that the shell is a monomolecular layer of native and 
denatured albumin in multiple orientations. The albumin shell is held together through disulfide bonds between cystein residues formed during cavitation. Covalent cross-linking may explain the relative rigidity of albumin shells observed during ultrasonic insonification ${ }^{7}$.

\section{METHOD OF LOADING DRUG INTO MICROBUBBLE}

Designing microbubbles that can carry cargo with high efficiency and high target-specificity is

particularly important for minimizing drug dosage, potentially reducing adverse side-effects, and lowering the expense of treatment.

Albumin-based microbubbles can potentially incorporate large amounts of plasmid DNA within the thick protein shell, although this remains to be verified. While this may improve the loading capacity of the microbubbles, the release of the shellincorporated pDNA (or other drugs) needs to be studied further. Complete breakdown of the shell of microbubbles is a short time during insonification would be required to release all of the incorporated cargo, which may not occur with protein-based microbubbles. Promoting adhesion of the polymer based microbubbles to the endothelial lumen where they can release their cargo over longer periods of time may significantly improve their functionality as drug/gene delivery vehicles ${ }^{7}$.

Lipid-based microbubbles, which can rapidly break apart, may be a more favorable in terms of designing rationale vectors for drug/gene delivery. Chemically linking the carriers to the surface of the microbubbles can dramatically improve the surface area for DNA loading and improve transfection properties of DNA after ultrasound mediated release. This novel method of formulation is a recent and promising avenue of research but still requires in vivo testing to demonstrate efficacy ${ }^{8}$.

\section{USE OF MICROBUBBLES}

The non viral delivery of vehicles, such as plasmids and antisense oligonucleotides, has been associated with a lower transfection efficiency and transient expression of the gene product. The first published report of targeted DNA delivery was performed in 1996, using surface ultrasound and intravenously delivered microbubbles carrying antisense oligonucleotides. In 1997, Bao et al described the use of ultrasound and albumin-coated microbubbles to enhance the transfection of luciferase reporter plasmid in cultured hamster cells. Since then, many studies have confirmed the efficacy of ultrasoundmediated microbubble destruction for drug and gene delivery, both in vitro and in vivo ${ }^{9}$.

Shohet et al demonstrated for the first time with an adenovirus vector that the ultrasound-mediated disruption of gas-filled microbubbles could be used to direct transgene expression to the heart in vivo. They showed that intravenously injected recombinant adenovirus vectors encoding a beta-galactosidase reporter gene were successfully delivered to normal rat myo- cardium using microbubbles and transthoracic $1.3 \mathrm{MHz}$ diagnostic ultrasound, at a mechanical index of 1.5 , delivered at a burst of 3 frames of ultrasound every 4 to 6 cardiac cycles ${ }^{10}$.

\section{CONCLUSION:}

Most of the focus on developing more effective microbubble formulations has been placed on improving drug carrying capacity and targeted release. There are still other potential barriers to drug/gene delivery efficiency that need to be addressed. Currently, most in vivo research focuses on targeting tumors or other organs with fenestrated endothelial lining. In order to expand on the usefulness of microbubbles in biomedical applications, more focus needs to be placed on designing more efficient drug carriers to promote intracellular uptake and drug transport. The potential utility of microbubbles in biomedical applications is continually growing as novel formulations and methods emerge. Microbubbles provide a unique range of responses to ultrasound, which makes them useful for contrast ultrasound imaging, identifying molecular expression and targeting drugs to specific tissue sites. Advances in our understanding of the underlying physicochemical properties has led to a recent burst in the development of novel constructs, including the use of bimodal brushes, polyelectrolyte multilayer, surface compartmentalization, and nanoparticle- microbubble hybrids. Microbubbles are 
both an art and a science. There's no way to do it, and each new application provides a fresh challenge.

\section{REFERENCES:}

1. Lindner, J.R., Song, J., Christiansen, J., Klibanov, A.L., Xu, F, Ley, K., 2001. Ultrasound assessment of inflammation and renal tissue injury with microbubbles targeted to P-selectin. Circulation. 104, 2107-2112.

2. Weller, G.E., Lu, E., Csikari, M.M., Klibanov, A.L., Fischer, D., Wagner, W.R, 2003. Ultrasound imaging of acute cardiac transplant rejection with microbubbles targeted to intercellular adhesion molecule-1.Circulation. 108, 218-224.

3. Christiansen, C., Kryvi, H., Sontum, P.C.,1994. Biological effects in Diagnostic ultrasound. Skotland T. Biotechnology and Applied Biochemistry. 19, 307-320.

4. Verder, H., Ebbesen, F., Linderholm, B., Robertson, B., Eschen, C., Arroe, M., Lange, A., Grytter, C., Bohlin, K., Bertelsen, A., Swedish, D.G.,2003. Prediction of respiratory distress syndrome by the microbubble stability test on gastric aspirates in newborns of less than 32 weeks' gestation. Multicentre Study. Acta Paediatr. 92,728733.

5. Cavalieri, F., Hamassi, A., Chiessi, E,, Paradossi, G., 2005. Stable polymeric microballoons as multifunctional device for biomedical uses: synthesis and characterization. Langmuir. 21, 8758-8764.
Solving these riddles requires experience, skill and the mastry of many different technologies

6. Borden, M.A., Martinez, G.V., Ricker, J., Tsvetkova, N., Longo, M., Gillies, R.J., Dayton, P.A., Ferrara, K.W., 2006. Lateral phase separation in lipid-coated microbubbles. Langmuir. 22, 42914297.

7. Miller, M.W., 2000. Microbubble- enhanced ultrasound for vascular gene delivery. Gene transfection and drug delivery. 26, S59-S62.

8. Unger, E.C., McCreery, T.P., Sweitzer, R.H., Caldwell, V.E., Wu, Y.,1998. Acoustically active lipospheres containing paclitaxel: a new therapeutic ultrasound contrast agent. Invest Radiol. 33,886-892.

9. Bao, S., Thrall, B.D., Miller, D.L., 1997. Transfection of a reporter plasmid into cultured cells by sonoporation in vitro. Ultrasound Med Biol. 23, 953-959.

10. Bekeredjian, R., Chen, S., Frenkel, P.A., Grayburn, P.A., Shohet, R.V., 2003.Ultrasoundtargeted microbubble destruction can repeatedly direct highly specific plasmid expression to the heart. Circulation. 108, 1022-1026.

11. Shohet MD., Raffi Bekeridijan MD., Paul A Grayburn MD. 2005. Use of ultrasound contrast agents for gene or drug delivery in cardiovascular medicine. J. of Amer. Col. of Card. 45, 329-335. 